\title{
TENSIONES ENTRE EL PATRIMONIO TANGIBLE E INTANGIBLE EN YUCATÁN, MÉXICO: LA IMPOSIBILIDAD DE RE-CREAR UNA CULTURA SIN ALTERAR SUS CARACTERÍSTICAS*
}

\author{
TENSIONS BETWEEN TANGIBLE AND INTANGIBLE HERITAGE IN YUCATÁN, \\ MEXICO: THE IMPOSSIBILITY OF RE-CREATING A CULTURE WITHOUT \\ ALTERING ITS CHARACTERISTICS
}

\author{
Fernando Armstrong-Fumero ${ }^{1}$
}

\begin{abstract}
Este artículo examina las tensiones y diálogos entre el patrimonio cultural tangible e intangible a través del caso del reciclaje arquitectónico en Yucatán, México. Ciertas contradicciones son evidentes en las definiciones oficiales de estos dos tipos de patrimonio en declaraciones que caracterizan al patrimonio intangible como algo dinámico y activo y al patrimonio tangible como una serie de objetos que se deben mantener in situ sin alteraciones humanas. Estas tensiones son aún más evidentes cuando las leyes que rigen el patrimonio cultural se aplican dentro de las comunidades rurales mayahablantes, cuyos residentes participan en una serie de tradiciones de colonización y reciclaje arquitectónico que son irreconciliables con la visión de un patrimonio tangible inalterable. La tensión inherente entre estas perspectivas hacia el paisaje y los restos prehispánicos se podría usar como la base para elaborar formas pragmáticas de colaboración e inclusión.
\end{abstract}

Palabras claves: patrimonio intangible, Yucatán, Maya, conservación.

This article examines tensions and dialogs between tangible and intangible cultural heritage through the case of architectural recycling in Yucatán, Mexico. Certain contradictions are evident in the official definitions of these two types of heritage, which characterize intangible heritage as something dynamic and active and it tangible heritage as a series of objects that must be maintained in situ without human alteration. These tensions are even more evident when heritage laws are applied inside of Maya-speaking communities, whose residents participate in a tradition of colonization and architectural recycling that seems irreconcilable with the vision of tangible heritage as unalterable. The inherent tension between these two perspectives on landscape and pre-Hispanic ruins can be the basis for developing pragmatic forms of collaboration and inclusion.

Key words: Intangible heritage, Maya, Yucatán, conservation.

Declaraciones de la UNESCO y otras instituciones internacionales plantean a la promoción del patrimonio cultural intangible como una contraparte del manejo de los objetos de patrimonio tangible y material. En muchos casos, estos dos enfoques han funcionado de forma complementaria dentro de proyectos que buscan adecuar la investigación arqueológica a los intereses de los descendientes de las sociedades antiguas que estudian. Pero en otros casos, la lógica institucional de la protección y custodia de restos arqueológicos genera tensiones con las prácticas y creencias que encarnan al patrimonio intangible de las comunidades locales.
En este ensayo examinaré estas tensiones a través de una tradición polémica del pueblo mayahablante de Yucatán: el uso "destructivo" de materiales excavados de edificios prehispánicos en la construcción de habitaciones modernas. El reúso de materiales excavados de sitios prehispánicos ha sido un elemento clave de las tradiciones de arquitectura vernácula en Yucatán. Más que un uso pragmático de materiales preelaborados, la manipulación de elementos antropogénicos en el paisaje forma parte de un conjunto de territorialidades y memoria colectiva en el cual la reocupación y rehabilitación de espacios previamente abandonados tiene gran

* Artículo seleccionado del conjunto de ponencias presentadas en el Simposio Shifting from Object-centered Research to People-focused Application: Current Approaches to Public Archaeology from Latin America and the Caribbean, realizado en el marco de la 75 reunión anual de la Sociedad de Arqueología Americana, Saint Louis, Missouri, EUA, abril 2010. Este manuscrito fue evaluado por investigadores externos y editado por Reiko Ishihara-Brito y Cameron Griffith, en su calidad de editores invitados de la Revista.

1 Departamento de Antropología, Smith College, 10 Prospect Street, 202 Northampton, MA, EUA. farmstro@ smith.edu 
importancia. Aunque esta dimensión del patrimonio intangible del pueblo Maya Yucateco parece contradecir estatutos federales e internacionales que promueven la preservación de restos prehispánicos in situ, también nos ayuda a reflexionar sobre las bases conceptuales y cultura jurídica que facilitarían el desarrollo de una arqueología inclusiva.

Mi análisis de este fenómeno se basa en un proyecto sobre las interacciones entre los pueblos mayahablantes y el paisaje que Julio Hoil y yo iniciamos en la comunidad de Xcalakdzonot en 2007. Es importante resaltar que, aunque esta investigación implica una mirada crítica a las prácticas de la arqueología académica y oficial, el tono de las discusiones que hemos tenido con nuestros colaboradores en la comunidad de Xcalakdzonot no ha sido uno de rechazo a leyes vigentes sobre el patrimonio cultural tangible. Nos hemos asegurado de que nuestro trabajo esté conforme con todos los estatutos del Instituto Nacional de Antropología e Historia (INAH), y hemos conversado sobre la utilidad que se puede derivar de una colaboración más estrecha entre gente de la comunidad e instituciones federales. Uno de los principales éxitos de nuestro proyecto ha sido el hecho de desarrollar un espacio en el cual la gente de Xcalakdzonot ha expresado su interés en colaborar con las instituciones oficiales a la vez que expresan sus propios intereses y perspectivas.

Además, debo agregar que la reutilización de materiales prehispánicos no parece haber sido practicada en forma extensiva en los últimos diez años, ya que el bloque de cemento y otros materiales industriales se han hecho más disponibles dentro de la comunidad. En este sentido, la tradición de reciclaje arquitectónico encarna formas de interactuar con elementos antropogénicos en el paisaje que son típicos de la cultura rural yucateca, pero no es necesariamente una actividad que sea común hoy en día. Aun así, esta tradición representa un punto particularmente interesante para explorar formas de diálogo entre el manejo del patrimonio tangible e intangible en comunidades como Xcalakdzonot, dado que es una práctica que tenía gran importancia para agricultores mayahablantes hasta hace muy poco, pero que parece contradecir las metas fundamentales de instituciones como el INAH.

El reciclaje arquitectónico y otros elementos de la percepción y memoria del paisaje por los agricultores mayahablantes muestran un elemento clave de la experiencia histórica de esta zona: la movilidad. A través de nuestras investigaciones de historia oral, fuentes documentales y exploraciones preliminares del ejido de la comunidad, hemos documentado varias olas de asentamientos y abandono en los períodos prehispánicos, coloniales y modernos en el espacio que actualmente corresponde a la comunidad de Xcalakdzonot. Estos patrones de asentamiento, abandono y reocupación culminaron en la fundación del pueblo actual entre 1900 y 1929 por agricultores que emigraron de otras comunidades, pero que traían un conocimiento previo de los recursos naturales y antropogénicos de las tierras que estaban colonizando. En este contexto histórico, las evidencias del reciclaje arquitectónico le dan forma tangible a la continuidad histórica de una serie de conocimientos que fueron transmitidos por narrativas orales durante muchas generaciones, preservando los nombres de lugares aun durante períodos en que no fueron poblados. Mientras que este conjunto de conocimientos y habitus (Bourdieu 1977) espacial forma una parte integral del conjunto de prácticas tradicionales que definen el patrimonio intangible del pueblo yucateco, éste implica que haya ciertas tensiones con algunos de los preceptos fundamentales que rigen las leyes para la defensa del patrimonio arqueológico. Estas tensiones también son evidentes en las distintas tradiciones jurídicas que contribuyeron al origen de reglamentos sobre patrimonio tangible e intangible, que será el enfoque de la próxima sección.

\section{Tensiones entre Patrimonio Tangible e Intangible}

La noción de patrimonio cultural intangible toma mayor importancia política a partir de los años 1990 del siglo XX, como parte de un movimiento internacional que definió a la defensa de las herencias culturales e identidades de pueblos indígenas o tribales como un derecho básico en sociedades democráticas. En este sentido, forman parte de una serie de declaraciones y reglamentos internacionales que surgieron al fin de la Guerra Fría, como la declaración 169 de la Organización Internacional del Trabajo, que redefinieron a la identidad y autonomía étnica en términos de derechos humanos (Ramos 2002; Yashar 2005). En México, estos decretos internacionales han sido incorporados en una serie de reformas, incluyendo las constituciones políticas de varios estados y de la federación, respecto a los derechos de pueblos indígenas (López Bárcena 2003).

El hecho de que este complejo de patrimonio intangible, identidad indígena y derechos humanos solo surgió en las últimas tres décadas presenta un 
contraste a la historia de instituciones nacionales que han manejado la custodia del patrimonio tangible. El actual Instituto Nacional de Antropología e Historia de México fue establecido en 1941 sobre una tradición jurídica con raíces en el siglo XIX. Leyes promulgadas en los años 1850 y 1860 tendieron hacia el establecimiento de los monumentos arqueológicos como propiedad del Estado, un hecho que se estableció explícitamente con un decreto en 1897. Desde la creación de la Inspección de Monumentos Arqueológicos de la República en 1875 , instituciones federales han coordinado trabajos de investigación, restauración y custodia que establecen la condición del patrimonio tangible prehispánico e histórico como dominio eminente del Estado mexicano (Bernal 1980:140; Breglia 2006).

Aunque las tensiones entre la definición institucional del patrimonio arqueológico y la identidad territorial de comunidades mayahablantes han sido bien documentadas por estudios realizados por Quetzil Castañeda (1996) y Lisa Breglia (2006), algo que quisiera resaltar aquí es una tensión dentro de los mismos discursos sobre patrimonio tangible e intangible que son promulgados por las instituciones oficiales. Una declaración emitida por UNESCO en 2003 plantea al patrimonio intangible como algo que es "constantemente recreado por las comunidades y los grupos como una respuesta a su medio ambiente, sus interacciones con la naturaleza, y su historia" (UNESCO 2003). El reúso de materiales arquitectónicos en Yucatán contribuye a estos procesos de "recreación", a través de la rehabilitación de lugares dentro del paisaje yucateco que fueron abandonados y repoblados en el transcurso de varias generaciones. Pero es precisamente ese elemento de la arquitectura vernácula de Yucatán que entra en conflicto con leyes que nombran al Estado como el único ente con el derecho legítimo de alterar la forma física de los restos prehispánicos dentro de los confines jurisdiccionales de la federación mexicana. Tanto las leyes nacionales como la mayoría de los códigos internacionales caracterizan al reciclaje arquitectónico como un proceso destructivo. La Convención de 1972 de UNESCO sobre el patrimonio de la humanidad cita a la extensión de habitación humana como una de las principales amenazas a los sitios arqueológicos que se han designado como "apeligrados". Esta caracterización de la preservación del patrimonio como un "rescate" de la actividad humana también domina los códigos legales implementados por el INAH (1975). La construcción en gran y pequeña escala, sea en propiedad privada o colectiva, debe cumplir con estatutos que prohíben la "alteración de las características" de estructuras que han sido designadas como monumentos por estudios académicos y decretos federales.

Este contraste es evidente en las interacciones entre arqueólogos y los residentes de comunidades como Xcalakdzonot, particularmente cuando se toma en cuenta la temporalidad que estas dos tradiciones le atribuyen al patrimonio tangible e intangible. Es decir, las definiciones oficiales de patrimonio intangible caracterizan a éste en términos procesuales como algo que es "constantemente recreado", mientras que los códigos de patrimonio tangible plantean un momento de ruptura histórica después del cual cualquier actividad humana puede representar una "alteración de las características" que afecta el valor del objeto. Como veremos, esta tensión entre la temporalidad del patrimonio tangible e intangible es particularmente evidente en el contexto de los patrones de asentamiento en el Yucatán rural. Una definición sincrónica -o anacrónica- del objeto de patrimonio tangible es algo muy distinto a la praxis doméstica de una comunidad mayahablante.

\section{Transformaciones de Paisajes y Espacios Domésticos}

Como mencioné anteriormente, una de las experiencias distintivas de la población mayahablante del oriente de Yucatán ha sido la movilidad. En parte, esto se debe a las condiciones ecológicas de las tierras bajas del norte de la zona maya, donde ha predominado una agricultura de tumba y roza, en la cual un campo de cultivo es abandonado después de dos o tres temporadas de actividad productiva. Otros factores de la historia social de la región, como el movimiento de pueblos indígenas a zonas de refugio en el sur de la península durante el período colonial (Bracamonte y Sosa 2001; Jones 1989), o el desplazamiento de gran parte de la población regional después de la "Guerra de Castas" de 1847 (Dumond 1997), han motivado migraciones de gran y pequeña escala por siglos. Esta movilidad ha contribuido al desarrollo de una identidad distintiva entre los yucatecos mayahablantes, particularmente en la zona del estado donde se halla Xcalakdzonot. Como otros pueblos mayahablantes en Chiapas y las tierras altas de Guatemala, los yucatecos rurales conservan el uso vigoroso de una lengua indígena y una cosmovisión con elementos de origen 
prehispánicos. Pero donde hablantes de Maya-Tzotzil, como son los habitantes de San Juan Chamula en Chiapas, cuentan historias que sitúan a la fundación de sus comunidades en tiempo mitológico (Gossen 1996), todos los cuentos que hemos grabado sobre los orígenes de Xcalakdzonot se refieren a eventos que tomaron lugar en los años 1920, cuando el pueblo fue dotado de tierras ejidales por el gobierno federal mexicano. Los conocimientos del paisaje tienen una gran profundidad histórica, y forman parte importante de la identidad colectiva de los habitantes de Yucatán. Pero esa misma historia oral muestra el hecho que muchas de las comunidades (kaajo'ob en Maya) que existen hoy en día son producto de etapas recientes en una historia más larga de asentamiento y migración.

Esta tradición de movilidad ha influido la cultura material y habitus espacial de la sociedad yucateca mayahablante en varias formas. Existen paralelos interesantes entre los patrones de asentamiento que ocurren en el transcurso de varias generaciones y las prácticas que ocurren en una escala temporal menor dentro de los grupos domésticos o "solares". Un aspecto que ha figurado en nuestras investigaciones son las formas de reúso y refuncionalización que ocurren dentro de los solares en pueblos como Xcalakdzonot $^{1}$. Estos son conjuntos de edificios habitacionales y de actividad económica muy dinámicos, cuyas estructuras están hechas de materiales con varios grados de permanencia y que dejan distintas huellas en el mundo material. Mientras que muchas habitaciones del período prehispánico fueron construidas sobre plataformas de piedra y escombros de más de dos metros, el uso de piedra en las estructuras domésticas que fueron construidas desde el período colonial tiende a ser reducido a un basamento bajo y una muralla o $k$ 'oot que rodea los límites del solar. Aunque se están construyendo más y más edificios de bloc industrial, el cuerpo de la mayoría de las casas en Xcalakdzonot y los pueblos vecinos consiste de postes de maderas duras techadas con palma de guano. Diferentes elementos de este complejo son renovados a distintas escalas temporales. Partes del techo son reparadas cada año, mientras que los postes más anchos de la superestructura de una casa pueden durar una década o más. Murallas y basamentos del período colonial y siglo XIX aún se pueden registrar arqueológicamente, y en algunos casos han sido usados como base para varias olas distintas de asentamiento.
Los distintos ciclos de renovación de los materiales que componen a un grupo doméstico también implican que algunos elementos arquitectónicos son reciclados cuando se altera la organización espacial de un solar. Por ejemplo, los cuatro postes de madera conocidos como okom, piezas claves que soportan el peso entero del techo de una casa, son tan masivos y están enterrados en el suelo a tal profundidad, que son extremadamente difíciles de sacar cuando una estructura es abandonada o destruida. En muchos casos, se dejan en su posición original. Dependiendo de la dureza de las maderas que se usen para elaborar un okom, estos pueden perdurar muchos años en los espacios domésticos y públicos. Allí sirven una serie de funciones secundarias, como los que están siendo usados como tendederos de ropa en la Figura 1. El okom de la Figura 2 proviene de Popolá, una comunidad hacia el oeste de Xcalakdzonot, donde fue parte de una casa que se desmontó para crear espacio cuando se aprobaron fondos federales para hacer un parque en el centro del pueblo en los años 1990. El okom quedó in situ donde sirvió como un poste para pintar o pegar anuncios de diferentes campañas políticas u otros eventos. Lo que ejemplos como estos muestran es que la idea de que los restos de una estructura vieja pueden o deben existir sin la "alteración de sus características" representa una lógica muy distinta a la de la vida cotidiana de un pueblo como Xcalakdzonot.

El tipo de refuncionalización que puede convertir a un okom en tendedero o rótulo político también se aplica a los restos materiales de asentamientos antiguos cuando los agricultores fomentan nuevos núcleos de población. Los procesos de renovación y refuncionalización que ocurren en el solar pueden servir como un modelo para procesos que ocurren en mayor escala espacial y temporal en los paisajes que son utilizados para la agricultura de tumba y roza. Las categorías tradicionales del paisaje Maya Yucateco-kaaj o pueblo, kool o milpa, y k'aax o monte (Quintal et al. 2003)- no representan cualidades fijas de los espacios, sino diferentes etapas en el uso humano de sitios dentro de un paisaje. La transformación de un lugar en kaaj, k'aax o kool se debe en gran parte a la temporalidad de la agricultura de tumba y roza. Después de varios años de uso, un kool es abandonado y revierte en k'aax. Este mismo patrón de agricultura también puede motivar movimientos de la población. En muchos casos, la necesidad de cultivar nuevos campos conduce a los 


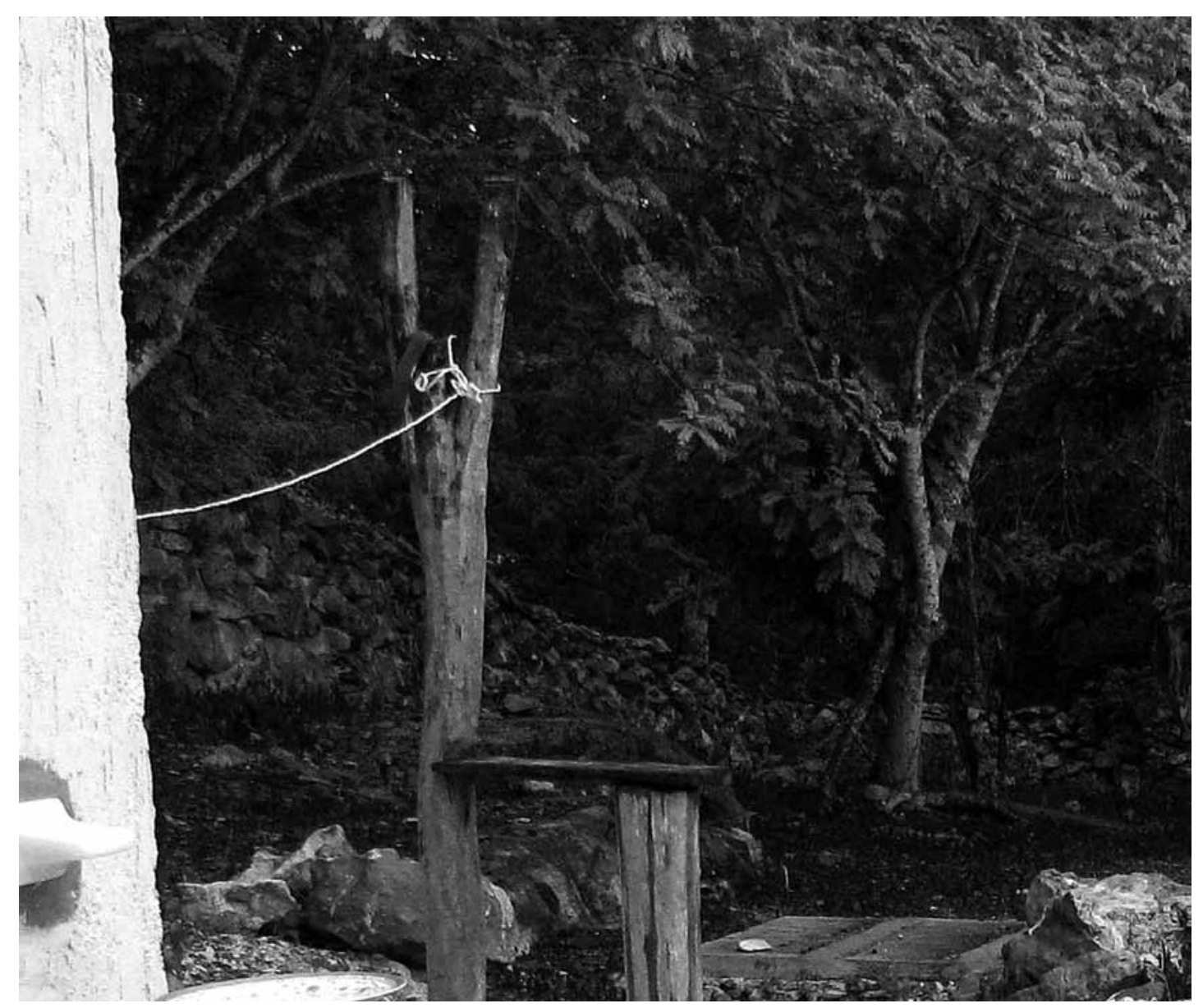

Figura 1. Un okom transformado en tendedero de ropa.

An okom transformed into a clothesline.

agricultores a sembrar kool lejos del kaaj, cosa que puede motivar el establecimiento de asentamientos permanentes o semipermanentes conocidos como kaajtal. Hasta principios del siglo XX, estos asentamientos podían eventualmente llegar al estatus de kaaj, mientras que muchos más fueron abandonados y se revirtieron en k'aax.

Estos ciclos de expansión, fomento de nuevos asentamientos y abandono, no son sólo una reacción al medio ambiente, sino también algo que puede ser iniciado o acelerado por conflictos dentro de comunidades existentes. Durante el período colonial, la migración fue una técnica utilizada por pueblos indígenas para preservar un grado de autonomía frente al dominio español (Bracamonte y Sosa y Solís Robleda 1996). La historia oral de comunidades como Xcalakdzonot, tanto como textos que documentan la distribución de tierras ejidales en el siglo XX (Armstrong-Fumero 2007), indican que la división de comunidades y fundación de nuevos asentamientos siguió siendo común en casos de conflictos internos en pueblos rurales.

Un resultado de la reproducción de estos patrones de cultivo y asentamiento a través de las generaciones es el hecho que el k'aax de Yucatán no es necesariamente un espacio salvaje o silvestre, sino un espacio en descanso o barbecho que está lleno de lugares nombrados que fueron ocupados en el pasado y que siguen siendo posibles bases para nuevos asentamientos. Uno de los artefactos intangibles de estos procesos de migración, asentamiento y abandono es la memoria colectiva de los nombres de sitios en el paisaje que, en diferentes momentos de la historia, existieron como k'aax, kaaj, o kool. El monte yucateco está repleto de miles de lugares nombrados que tienen cenotes, pozos, 


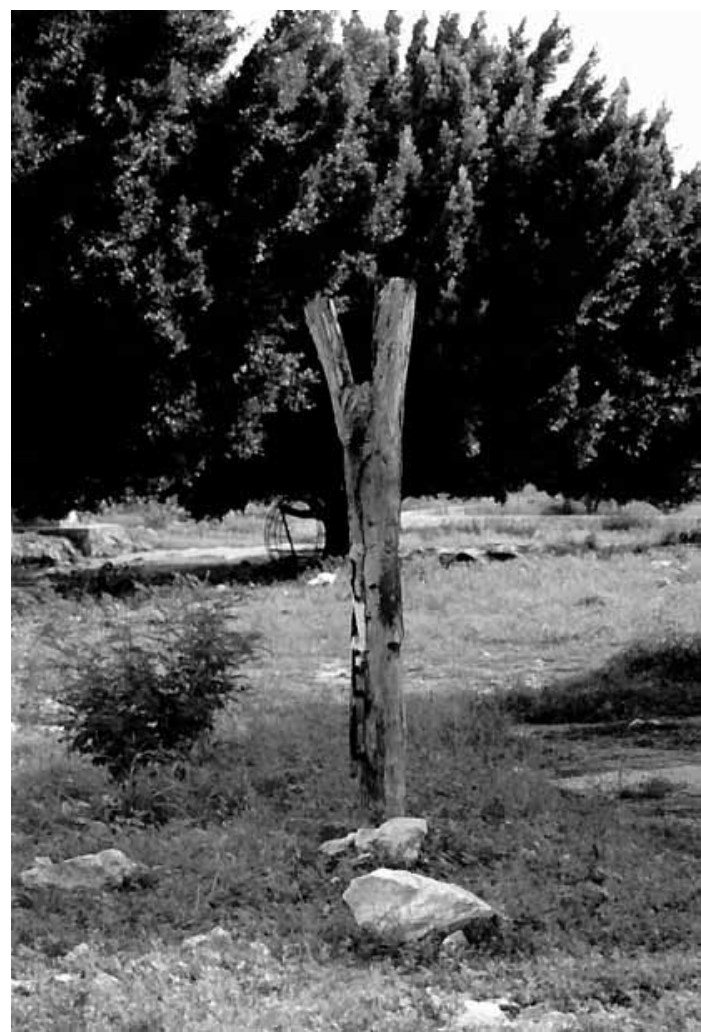

Figura 2. Un okom en que se pintó propaganda política para el candidato Israel Palma Santos.

An okom on which political propaganda for the candidate Israel Palma Santos was painted.

rejolladas y suelos de diferentes calidades. El aspecto descriptivo de la toponimia maya es evidente por los nombres de muchos pueblos -como Xcocail, "el sitio de las luciérnagas", Ticimul "montículo seco", Xcalakdzonot "dos cenotes gemelos"- que conmemoran detalles del paisaje natural y antropogénicos que existían antes de la fundación de una comunidad permanente. Muchos de los sitios que los agricultores de Xcalakdzonot pueden nombrar hoy día-como Siete Pilas (nombrado por una serie de trojes de ganado que fueron hechos de piedras prehispánicas) y Xtakejil ("lugar de excremento de venado")- no han sido poblados en varias generaciones. Pero aun así, se conocen a través de la historia oral y por los recorridos del k'aax que se hacen en la cacería o en busca de recursos como madera y plantas medicinales. Muchos de estos topónimos -como el cenote Akulá en Xcalakdzonotaparecen en documentos escritos en maya y español entre los siglos XVI y XVIII (Julio Hoil Gutiérrez comunicación personal 2010). El hecho de que hayan conformado parte del conocimiento de generaciones de campesinos, quienes en su mayoría no hubieran sabido leer o escribir hasta el siglo XX, muestra la fidelidad con que la memoria colectiva e historia oral pueden preservar estos conocimientos del paisaje a través de los siglos.

Aquí podemos ver el contexto histórico y sociocultural de las prácticas de reciclaje arquitectónico. Los mismos factores que han guiado los patrones de asentamiento también han determinado donde existen aglomeraciones de restos arquitectónicos de distintas etapas históricas. Dada la hidrografía de la península de Yucatán, donde las únicas fuentes de agua son los cenotes o pozos naturales, ciertos lugares provechosos han atraído múltiples olas de población, a veces separadas por décadas o siglos. Después de repetidos ciclos de asentamiento, despoblamiento y reocupación, lugares como los cenotes tienden a estar rodeados por los restos arquitectónicos de asentamientos prehispánicos, coloniales y modernos. Para todas estas olas de pobladores, la disponibilidad de piedras pre-formadas en las ruinas de asentamientos anteriores contribuía al atractivo de estos lugares.

Como una experiencia cotidiana, el reúso de materiales prehispánicos suele repetir los mismos patrones de refuncionalización que ocurre dentro de los solares. Como el okom que se transforma de columna arquitectónica a tendedero, artefactos de asentamientos abandonados cerca de un cenote $\mathrm{u}$ otro atractivo natural pueden ser refuncionalizados por nuevos pobladores. Algo que es común en toda la península de Yucatán es el uso de metates prehispánicos como trojes de ganado (Figura 3). A través de esta tradición, objetos que fueron utilizados en la producción de alimentos en asentamientos prehispánicos sirven para apoyar una economía contemporánea que aprovecha de los mismos recursos hidrológicos.

\section{Implicaciones para la Arqueología}

Como un fenómeno sociocultural que ha formado parte de la vida cotidiana de generaciones de agricultores mayahablantes, la memoria colectiva del paisaje y sus diferentes ciclos de uso y desuso son un patrimonio intangible que ha contribuido a la fundación y sobrevivencia de comunidades como Xcalakdzonot. Pero, cuando las prácticas de reciclaje que forman parte de este patrimonio se aplican a restos prehispánicos, entran en un punto de tensión 


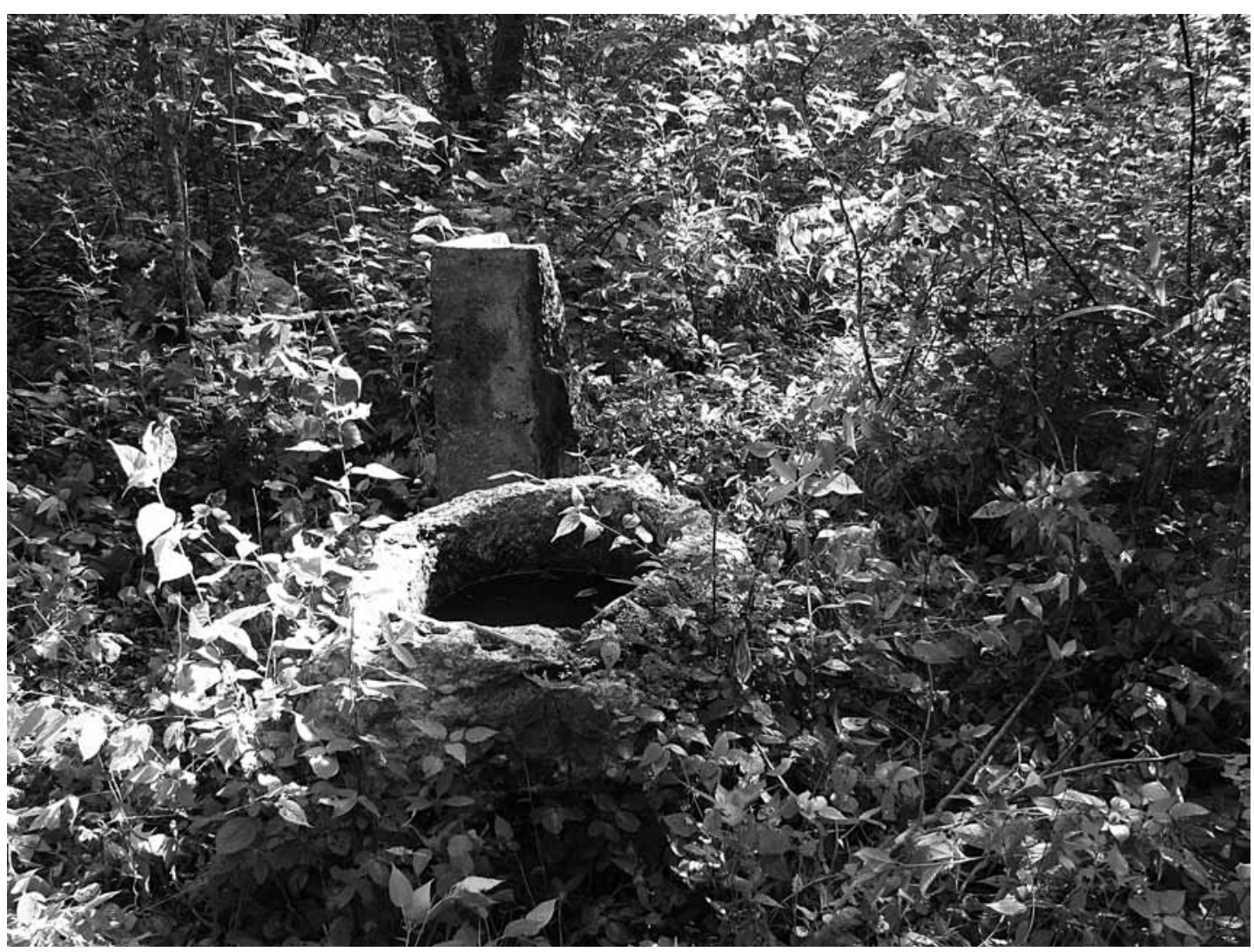

Figura 3. Un metate prehispánico que fue transportado a un pozo en ca. 1950, y que ha servido a generaciones de campesinos como un bebedero para sus caballos y vacas.

A pre-Hispanic metate that was transported to a well ca. 1950, and which has served generations of peasants as a place to water their horses and cattle.

irreconciliable con las tradiciones jurídicas y bases éticas que sostienen a la protección oficial del patrimonio tangible de la nación y humanidad. Es por eso mismo que representa un punto interesante para pensar en los límites de la arqueología inclusiva. Concluiré este argumento con dos avenidas de exploración que pueden surgir de esta tensión, una conceptual y otra más pragmática.

La exploración conceptual trata sobre la sustancia de la "inclusión" y el diálogo. Arqueólogos postprocesualistas y varios etnógrafos se han enfrentado a esta cuestión de inclusión con el concepto de multivocalidad: una ética que pretende democratizar el conocimiento arqueológico reconociendo el derecho de diferentes comunidades de narrar su propia versión del pasado y de los sitios (El-Haj 1998; Castañeda y Matthews 2008; Hodder 2003; Kane 2003). Pero mientras que una perspectiva "multivocal" le presta autoridad interpretativa a públicos más amplios, nos dice muy poco sobre los derechos de comunidades descendientes para manipular la forma física de los objetos. La percepción y memoria de los paisajes en un pueblo como Xcalakdzonot no es sólo una cuestión de narrar historias; se trata también de un habitus espacial que incluye la manipulación física de los sitios que han sido bases para asentamientos previos. En este sentido, el hecho de sugerir que los residentes de Xcalakdzonot apliquen leyes que prohíben la "alteración de las características" de una estructura prehispánica siempre implica una "alteración de las características" de las tradiciones locales que se pretende preservar como patrimonio intangible. Este tipo de encrucijada ética y conceptual es una parte inherente de los diálogos entre instituciones oficiales y comunidades locales que no se puede resolver de forma sencilla con la ética de la multivocalidad.

Una avenida más pragmática de exploración sería reconceptualizar el diálogo en términos que tengan una presencia más tangible en la vida 
cotidiana de las comunidades mayahablantes. Algo que es evidente en los estudios etnográficos es que muchos yucatecos rurales relacionan las formas de trabajo asociadas con la excavación, restauración y custodia de los restos arqueológicos con actividades tradicionales asociadas a la agricultura (ArmstrongFumero 2011; Armstrong-Fumero y Hoil Gutiérrez 2010; Breglia 2006). Esto ha sido muy evidente en nuestras conversaciones con gente en Xcalakdzonot, quienes se refieren a la posibilidad de un proyecto arqueológico como "trabajar" los montículos, o en Maya con los términos "meyaj” (trabajo) o "yutskintik" (mejorar). Estos son los mismos términos que se usan en referencia a la obra de transformar k'aax en kool o kaaj.

Esta continuidad entre el trabajo de arqueología y la experiencia colectiva de agricultura y manejo del paisaje es un punto clave para el diálogo entre la arqueología y las comunidades mayahablantes. Este diálogo puede ser tan sencillo como plantear la preservación de los restos arqueológicos con más sensibilidad hacia la perspectiva tradicional de la comunidad; reconociendo que los mismos elementos del paisaje que sus abuelos y padres usaron como una fuente de piedra pueden generar salarios u otros beneficios para la comunidad si se "trabajan" en colaboración con un proyecto arqueológico. Pero también es importante que los antropólogos integren los conocimientos que la gente de comunidades rurales obtienen en sus interacciones con ruinas prehispánicas al análisis de la historia de los paisajes que han permitido la sobrevivencia de sociedades indígenas por tantos siglos. Esto es algo que estamos explorando en Xcalakdzonot con una integración de trabajo arqueológico, etnográfico y documental que tiene pocos precedentes en los estudios de Yucatán. Más allá del estudio interdisciplinario de los patrones de asentamiento y usos del paisaje, consideramos que los fines de este proyecto sean generar datos sobre los usos del paisaje que definen un patrimonio cultural, que está igualmente encarnado en los conocimientos intangibles de las comunidades mayahablantes como en los restos tangibles de sus antepasados.

Agradecimientos: Quisiera agradecer a Reiko Ishihara, Cameron Griffin, y a los otros participantes en la mesa de la reunión del SAA cuyos trabajos están incluidos en esta edición, además de los evaluadores. Como siempre, le debo gracias a mi coinvestigador Julio Hoil Gutiérrez, y a todos nuestros colaboradores en la comunidad de Xcalakdzonot. Una serie de conversaciones con Héctor Hernández y Rani Alexander han enriquecido mi perspectiva sobre muchos de los procesos que figuran en este artículo. También le agradezco a Melisa García, una colega de Smith College, por comentarios en una versión anterior de este ensayo.

\section{Referencias Citadas}

Armstrong-Fumero, F. 2007. Before There Was Culture Here. Dissertación doctoral, Departamento de Antropología Cultural y Social, Universidad de Stanford, Stanford.

- - - 2011. Words and things in Yucatán: poststructuralism and the everyday life of Mayan multiculturalism. Journal of the Royal Anthropological Institute 17:63-81.

Armstrong-Fumero, F. y J. Hoil Gutiérrez 2010. Community heritage and partnership in Xcalakdzonot, Yucatán. En Handbook of Postcolonial Archaeology, editado por U. Rizvi y J. Lydon, pp. 391-397. Left Coast Press, Walnut Creek, en prensa.

Bernal, I. 1980. A History of Mexican Anthropology. Thames and Hudson, New York.

Bourdieu, P. 1977. Outline of a Theory of Practice. Cambridge University Press, Cambridge.

Bracamonte y Sosa, P. 2001. La Conquista Inconclusa de Yucatán: Los Mayas de la Montaña. CIESAS, México.

Bracamonte y Sosa, P. y G. Solís Robleda 1996. Espacios Mayas de Autonomía: El Pacto Colonial en Yucatán. UADY, Mérida.
Breglia, L. 2006. Monumental Ambivalence: The Politics of Heritage. University of Texas Press, Austin.

Castañeda, Q. 1996. In the Museum of Maya Culture: Touring Chichén Itzá. University of Minnesota Press, Minneapolis.

Castañeda, Q. y C. Matthews (eds.) 2008. Ethnographic Archaeologies: Reflections on Stakeholders and Archaeological Practices. Altamira Press, Lanham.

Dumond, D. 1997. The Machete and the Cross: Campesino Rebellion in Yucatán. University of Nebraska Press, Lincoln.

El-Haj, N.A. 1998. Translating truths: nationalism, the practice of archaeology, and the remaking of the past and present in contemporary Jerusalem. American Ethnologist 25:166-188.

Gossen, G. 1996. Telling Maya Tales: Tzotzil Identities in Modern Mexico. Routledge, New York.

Hodder, I. 2003. Sustainable time travel: toward a global politics of the past. En Politics of Archaeology and Identity in a Global Context, editado por S. Kane, pp. 139-147. Archaeological Institute of America, Boston. 
Instituto Nacional de Antropología e Historia (INAH, México) 1975. Reglamento de la Ley Federal Sobre Monumentos y Zonas Arqueológicas, Artísticos e Históricos. http://www.inah.gob.mx/ Transparencia/Archivos/207_regla_ley_fed_mntos_zon_arq. $p d f$ (14 de febrero).

Jones, G.D. 1989. Maya Resistance to Spanish Rule: Time and History on a Colonial Frontier. University of New Mexico Press, Albuquerque.

Kane, S. 2003. Politics of archaeology and identity in a global context. En Politics of Archaeology and Identity in a Global Context, editado por S. Kane, pp. 1-10. Archaeological Institute of America, Boston.

López Bárcena, F. 2005. Los Movimientos Indígenas en México: Rostros y Caminos. M.C. Editores, México.

Quintal, E.F., J. Bastarrachea, F. Briceño, M. Medina, B. Repetto, L. Rejón y M. Rosales 2003. U lu'umil maaya wíiniko'ob: la tierra de los mayas. En Diálogos con el Territorio: Simbolización sobre el Espacio en las Culturas Indígenas de México, editado por
A.M. Barabas, pp. 273-359. Instituto Nacional de Antropología e Historia, Ciudad de México.

Ramos, A.R. 2002. Cutting through state and class: sources and strategies of self-representation in Latin America. En Indigenous Movements, Self Representation and the State in Latin America, editado por K. Warren y J. Jackson, pp. 251-278. University of Texas Press, Austin.

United Nations Educational Scientific and Cultural Organization (UNESCO) 1972. Convention concerning the protection of the world cultural and natural heritage. http://whc.unesco.org/en/ conventiontext/ (14 febrero 2012).

- - - 2003. Convention for the safeguarding of intangible cultural heritage. http://www.unesco.org/culture/ich/index. php?lg=en\&pg=00006 (14 febrero 2012).

Yashar, D. 2005. Contesting Citizenship in Latin America: The Rise of Indigenous Movements and the Postliberal Challenge. Cambridge University Press, Cambridge.

\section{Nota}

1 La tesis doctoral del Prof. Héctor Hernández de la Facultad de Ciencias Antropológicas de la Universidad Autónoma de Yucatán incluirá un análisis detallado de la cultura material y los espacios domésticos en comunidades mayas modernas basadas en un estudio de la comunidad de Yaxuná. Mi propia perspectiva sobre los usos del espacio doméstico en Xcalakdzonot ha sido enriquecida por una serie de discusiones con Hernández. 
